\title{
Biosynthesis of an extracellular siderophore is essential for maintenance of mutualistic endophyte-grass symbioses
} C. R. VOISEY ${ }^{1}$, G. BRYAN ${ }^{1}$, I. LAMONT ${ }^{2}$ AND S. RASMUSSEN

${ }^{\prime}$ AgResearch Limited, Tennent Drive, Private Bag 11008, Palmerston North, New Zealand. ${ }^{2}$ Dept of Biochemistry, University of Otago, P.O. Box 56, Dunedin, New Zealand linda.johnson@agresearch.co.nz

\begin{abstract}
We have identified a novel siderophore from the mutualistic grass symbiont Epichloë festucae. Targeted gene replacement of a non-ribosomal peptide synthetase (termed sidF) from $E$. festucae eliminated biosynthesis of this extracellular siderophore. Structural characterisation, based on extensive mass spectrometry showed similarities to fusarinine-type siderophores. Plants inoculated with strains carrying a targeted deletion in $s i d F$ have a dramatic phenotype. Siderophore loss changed a mutualistic symbiont into an antagonistic fungus, causing de-regulation and proliferation of fungal hyphae, plant stunting and sometimes even tiller death. We hypothesise that loss of the biosynthesis of the siderophore disrupts iron homeostasis within the whole symbiotum, which is a critical factor for maintenance of mutualistic endophyte-grass symbioses.
\end{abstract}

Keywords: siderophore, iron homeostasis, NRPS

\section{Introduction}

Iron is an essential trace element required for nearly all living organisms. Due to its insolubility and toxicity in aerobic environments, iron is not freely available in living tissues. Under iron starvation, most micro-organisms synthesise siderophores low molecular weight, iron-chelating agents that solubilise iron (III) and control intracellular iron levels (Haas 2003; Renshaw et al. 2002). Some siderophores also function as virulence factors (Oide et al. 2006; Ratledge \& Dover 2000).

Endophytic fungi of the genus Epichloë (Ascomycota, Clavicipitaceae) and related asexual Neotyphodium species form mutualistic symbioses with temperate grasses (Poaceae). The symbiont's growth is confined to the intercellular spaces of leaf sheaths and blades. This symbiosis can be mutually beneficial and numerous biotic and abiotic advantages are conferred upon the host by the fungus (Schardl et al. 2004).

Most fungi produce hydroxamate-type siderophores (van der Helm \& Winkelmann 1994), which are typically composed of three hydroxamate groups linked by peptide or ester bonds to form an octahedral complex. Linkage of these groups is catalysed by non-ribosomal peptide synthetases (NRPS) which may also add additional amino acids (Plattner \& Diekmann 1994). These multifunctional enzymes are modular in structure whereby a typical module consists of a substrate adenylation domain, a peptidyl carrier domain, and a condensation domain that catalyses peptide bond formation (Finking \& Marahiel 2004). A few NRPS genes from filamentous fungi have been shown to encode NRPSs responsible for the synthesis of siderophores (Eisendle et al. 2003; Yuan et al. 2001; Oide et al. 2006; Tobiasen et al. 2006). Most notably, the NRPS encoded by NPS6 from Cochliobolus heterostrophus, Fusarium graminearum and Alternaria brassicicola (Oide et al. 2006) is responsible for biosynthesis of extracellular siderophores of the coprogen/fusarinine-type which was demonstrated to have a role in virulence to plants.

\section{Methods}

\section{Molecular biology}

An Epichloë festucae (FL1) fosmid library was constructed (Epicentre Biotechnologies). A gene replacement construct was created using Multisite Gateway Three-fragment Vector Construction Kit (Invitrogen). Entry clones were combined to produce a destination vector containing inserts of approximately $3 \mathrm{~kb}$ of 5 ' NRPS coding sequence, followed by a hygromycin resistant gene and approximately $3 \mathrm{~kb}$ of consecutive NRPS sequence. Protoplasts were prepared as described by (Tanaka et al. 2005and references within) and transformed using methodology of Vollmer \& Yanofsky (1986) and modifications by Itoh et al. (1994).

\section{Culture conditions}

Media for iron growth studies and chemical analyses were modified from (Mantle \& Nisbet 1976), with yeast extract replaced with $0.6 \mu \mathrm{M}$ thymine. Iron was omitted for iron-depleted media or supplemented with $\mathrm{FeSO}_{4}$ or $\mathrm{FeCl}_{3}$ as indicated.

\section{LCMSMS}

Samples were extracted from both culture supernatant and freezedried mycelia from iron-depleted and iron-supplemented liquid cultures, as described above, using the SPE method (McCormack et al. 2003). Analytes were eluted through a C18 Luna column (Phenomenex Torrence, CA, USA) $(150 \times 2 \mathrm{~mm}, 5 \mu \mathrm{m})$ at a flow rate of $200 \mu 1 \mathrm{~min}^{-1}$ using a Thermo Finnigan Surveyor HPLC system with a solvent gradient (solvent $\mathrm{A}: \mathrm{H}_{2} \mathrm{O} 0.1 \%$ formic acid; B: $\mathrm{MeCN} 0.1 \%$ formic acid), starting with $3 \%$ B, $97 \%$ A for 5 minutes and then increasing to $23 \% \mathrm{~B}$ over 15 minutes followed by a column wash at $95 \% \mathrm{~B}$. UV spectra were obtained using a Thermo surveyor PDA detector (200-600 nm) and monitored at

Figure 1 Modular organisation of E. festucae SidF, Aspergillus nidulans SidC and Ustilago maydis Sid2. A: adenylation domain, $P$ : peptidyl carrier protein, $C$ : condensation domain. $\mathrm{C}^{*}$ : non-functional condensation domain.
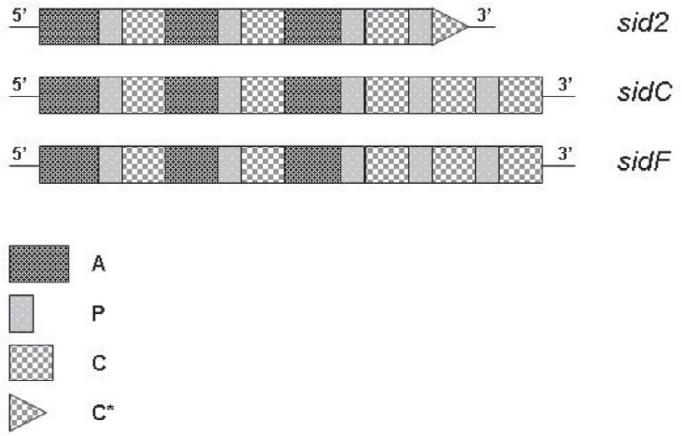
Figure 2 Comparison of wild-type (WT) and mutant ( $\Delta$ sidF) chromatograms from supernatants extracted from iron depleted (Fe-) or iron supplemented ( $\mathrm{Fe}+$ ) media. Peak at 14.7 represents the iron-free form of the siderophore and the peaks at 16.1 or 16.2 represents the iron-bound form.
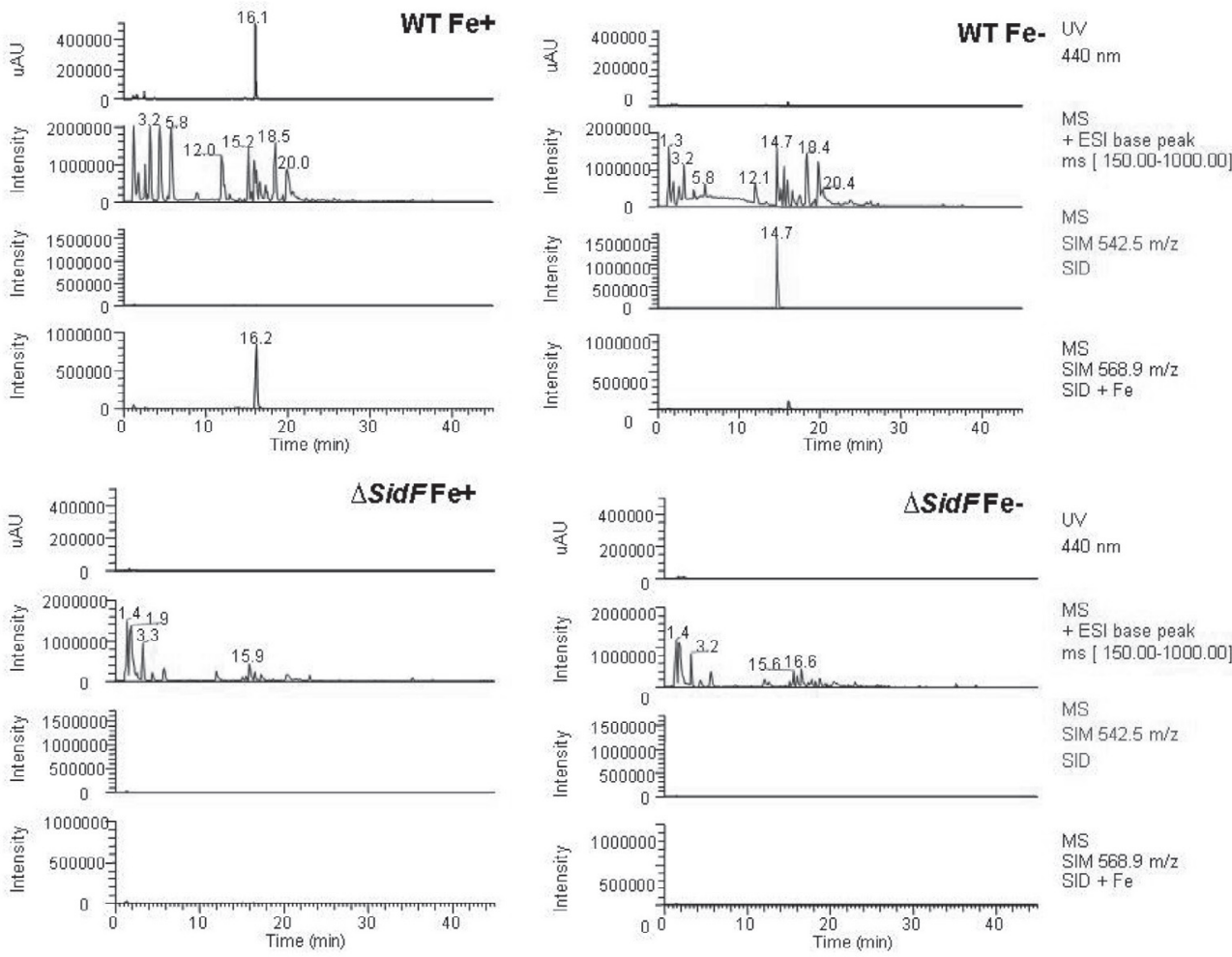

$440 \mathrm{~nm}$, which is typical for siderophore- $\mathrm{Fe}^{3+}$ complexes.

Mass spectra were determined with a linear ion trap mass spectrometer (Thermo LTQ) using ESI in +ve mode. The spray voltage was $5.0 \mathrm{kV}$ and the capillary temperature $275^{\circ} \mathrm{C}$. The flow rates of sheath gas, auxiliary gas, and sweep gas were set to 20,5 , and 10 (arbitrary units), respectively. Other parameters were optimised automatically by infusing ferrichrome in $\mathrm{H} 2 \mathrm{O}$ : MeCN:HCOOH $(75: 25: 0.1, \mathrm{v} / \mathrm{v} / \mathrm{v})$ at a flow rate of $200 \mu 1 \mathrm{~min}^{-1}$.

\section{Results}

A degenerate PCR strategy was undertaken to generate a library of endophyte NRPS sequences (Johnson et al. 2007). Characterisation of one of these NRPSs (termed sidF) is presented here. The promoter and entire open reading frame of sidF was obtained from an E. festucae fosmid library. A BlastX analysis of the deduced amino acid sequence revealed high identities to fungal NRPS genes involved in siderophore biosynthesis. The domain structure of sidF predicts the presence of three adenylation domains (Fig. 1).

To investigate whether sidF encodes a NRPS gene involved in siderophore biosynthesis, a gene replacement was performed by homologous recombination. Replacement mutants were selected and phenotypic growth characteristics were examined in vitro. On iron-depleted media supplemented with $100 \mu \mathrm{M}$ of bathophenanthrolinedisulfonic acid (an iron chelator), the wild-type strain's growth was not affected, but all mutant strains were unable to grow; a phenotype expected if no extracellular siderophores are synthesised. Addition of either $80 \mu \mathrm{M} \mathrm{Fe} 2+$ or

$\mathrm{Fe} 3+$ could overcome this growth deficiency.

To determine the metabolic end-product of $s i d F$, chemical analysis was carried out on both wild-type and knock-out strains. Both culture supernatant and mycelial extracts were analysed by LCMSMS coupled to PDA. Comparison of the wild-type and mutant chromatograms from the supernatant extractions clearly showed the absence of an extracellular siderophore in the mutant strains, indicating that $s i d F$ encodes an extracellular siderophore (Fig. 2). Extensive mass spectral studies, using LCMS $^{\mathrm{n}}$ and ICRTFMS (data not shown) indicated that this compound has three identical units consisting of a hydroxamate attached through the acyl group to anhydromevalonic acid and three additional amino acids. From our structural information, we believe the endophyte siderophore to be a novel member of the fusarinine family.

To find out if loss of sidF has an effect on the symbiotic relationship of $E$. festucae with perennial ryegrass, sidF mutant strains were inoculated into ryegrass seedling lines. Plants infected with siderophore mutant strains gave a surprisingly dramatic phenotype (Fig. 3). Microscopic examination of aniline-blue stained leaf sheaths from the mutant revealed on-going tip growth and excessive hyphal branching in the expanding leaf zone resulting in an increase in fungal biomass and loss of synchronisation of endophyte growth with that of its host plant. In addition, plant stunting and occasionally tiller death occurred. Transmission electron microscopy revealed an altered hyphal ultrastructure, including irregular hyphal shape and uncharacteristic vacuolation. 
Figure 3 Comparison of wild-type (WT) and individual siderophore minus mutants $(\triangle \operatorname{sidF})$ associated with perennial ryegrass.

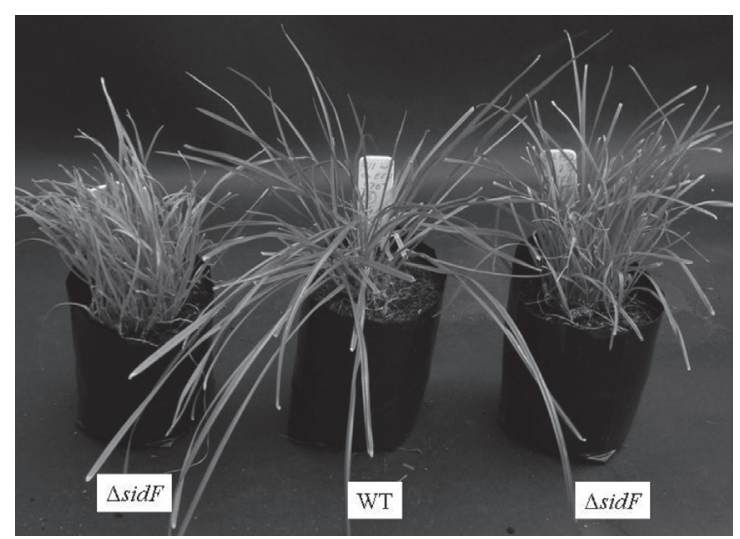

\section{Discussion}

No siderophores or genes involved in their biosynthesis have been reported previously from grass endophytes. We identified and characterised a fungal NRPS gene, sidF, from E. festucae and show that it is required for the biosynthesis of a novel hydroxamate siderophore, most likely of the fusarinine-type. Chemical analysis, combined with the inability of the $s i d F$ mutant to grow on iron-depleted media, indicates the presence of just one extracellular siderophore. Most significantly, associations of the siderophore mutant strains of the symbiotic endophyte with perennial ryegrass are detrimental and disrupt the symbiosis. In contrast, loss of extracellular siderophores through deletion of the NRPS gene NPS6 from fungal plant pathogens resulted in reduced virulence (Oide et al. 2006). We hypothesise that lack of the endophyte extracellular siderophore alters iron homeostasis of the symbiotum, perturbing the natural interaction and changing it from mutually beneficial to antagonistic.

\section{ACKNOWLEDGEMENTS}

We acknowledge the funding support from the New Zealand Foundation of Research Science and Technology, under contract AGRX0204.

\section{REFERENCES}

Eisendle, M.; Oberegger, H.; Zadra, I.; Haas, H. 2003. The siderophore system is essential for viability of Aspergillus nidulans: Functional analysis of two genes encoding Lornithine N5-monooxygenase ( $\operatorname{sidA}$ ) and a non-ribosomal peptide synthetase (sidC). Molecular Microbiology 49: 359.

Finking, R.; Marahiel, M.A. 2004. Biosynthesis of nonribosomal peptides 1. Annual Review of Microbiology 58: 453-488.

Haas, H. 2003. Molecular genetics of fungal siderophore biosynthesis and uptake: The role of siderophores in iron uptake and storage. Applied Microbiology and Biotechnology 62: 316 .
Itoh, Y.; Johnson, R.; Scott, B. 1994. Integrative transformation of the mycotoxin-producing fungus, Penicillium paxilli. Current Genetics 25: 508.

Johnson R.D.; Voisey C.; Johnson L.J.; Pratt J.; Fleetwood D.; Bryan G. 2007. Identification of NRPS gene families from Neotyphodium/Epichloë endophytic fungi that form mutualistic associations with cool season grasses. pp. 495

In: Proceedings of the 6th International Symposium on Fungal Endophytes of Grasses. Eds. Popay, A.J.; Thom, E.R. Grassland Research and Practice Series No. 13. New Zealand Grassland Association.

Mantle, P.G.; Nisbet, L.J. 1976. Differentiation of Claviceps purpurea in axenic culture. Journal of General Microbiology 93: 321.

McCormack, P.; Worsfold, P.J.; Gledhill, M. 2003. Separation and detection of siderophores produced by marine bacterioplankton using high-performance liquid chromatography with electrospray ionization mass spectrometry. Analytical Chemistry 75: 2647-2652.

Oide, S.; Moeder, W.; Krasnoff, S.; Gibson, D.; Haas, H.; Yoshioka, K.; Gillian Turgeon, B. 2006. NPS6, encoding a nonribosomal peptide synthetase involved in siderophoremediated iron metabolism, is a conserved virulence determinant of plant pathogenic ascomycetes. Plant Cell 18: 2836-2853.

Plattner, H.J. Diekmann, H. 1994. Enzymology of siderophore biosynthesis. pp. 99-116 In: Metal Ions in Fungi. Eds. Winkelmann, G. and Winge, D.R. Decker, New York.

Ratledge, C.; Dover, L.G. 2000. Iron metabolism in pathogenic bacteria. Annual Review of Microbiology 54: 881.

Renshaw, J.C.; Robson, G.D.; Trinci, A.P.J.; Wiebe, M.G.; Livens, F.R.; Collison, D.; Taylor, R.J. 2002. Fungal siderophores: structures, functions and applications. Mycological Research 106: 1123.

Schardl, C.L.; Leuchtmann, A.; Spiering, M.J. 2004. Symbioses of grasses with seedborne fungal endophytes. Annual Review of Plant Biology 55: 315-340.

Tanaka, A.; Tapper, B.A.; Popay, A.; Parker, E.J.; Scott, B. 2005. A symbiosis expressed non-ribosomal peptide synthetase from a mutualistic fungal endophyte of perennial ryegrass confers protection to the symbiotum from insect herbivory. Molecular Microbiology 57: 1036.

Tobiasen, C.; Aahman, J.; Ravnholt, K.S.; Bjerrum, M.J.; Grell, M.N.; Giese, H. 2006. Nonribosomal peptide synthetase (NPS) genes in Fusarium graminearum, F. culmorum and F. pseudograminearium and identification of NPS2 as the producer of ferricrocin. Current Genetics Online first.

van der Helm, D.; Winkelmann, G. 1994. Hydroxamates and polycarbonates as iron transport agents (siderophores) in fungi. pp. 39-148. In: Metal Ions in Fungi. Eds. Winkelmann, G.; Winge, D.R. Decker, New York.

Vollmer, S.J.; Yanofsky, C. 1986. Efficient cloning of genes of Neurospora crassa. Proceedings of the National Academy of Sciences of the United States of America 83: 4869.

Yuan, W.M.; Gentil, G.D.; Budde, A.D.; Leong, S.A. 2001. Characterisation of the Ustilago maydis sid2 gene, encoding a multidomain peptide synthetase in the ferrichrome biosynthetic gene cluster. Journal of Bacteriology 183: 4040. 